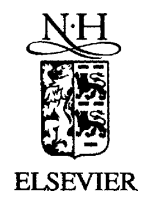

Information Sciences 141 (2002) 193-217

INFORMATION

SCIENCES

AN INIERVATTONAL JOURNAL

www.elsevier.com/locate/ins

\title{
Incremental class learning approach and its application to handwritten digit recognition
}

\author{
Jacek Mańdziuk $^{\mathrm{a}, *, 1}$, Lokendra Shastri ${ }^{\mathrm{b}}$

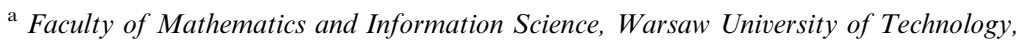 \\ Plac Politechniki 1, 00-661 Warsaw, Poland \\ b International Computer Science Institute, 1947 Center St., Suite 600, Berkeley, CA 94704, USA \\ Received 30 July 2001; accepted 27 September 2001 \\ Communicated by Subhash Kak
}

\begin{abstract}
Incremental class learning (ICL) provides a feasible framework for the development of scalable learning systems. Instead of learning a complex problem at once, ICL focuses on learning subproblems incrementally, one at a time - using the results of prior learning for subsequent learning - and then combining the solutions in an appropriate manner. With respect to multi-class classification problems, the ICL approach presented in this paper can be summarized as follows. Initially the system focuses on one category. After it learns this category, it tries to identify a compact subset of features (nodes) in the hidden layers, that are crucial for the recognition of this category. The system then freezes these crucial nodes (features) by fixing their incoming weights. As a result, these features cannot be obliterated in subsequent learning. These frozen features are available during subsequent learning and can serve as parts of weight structures built to recognize other categories. As more categories are learned, the set of features gradually stabilizes and learning a new category requires less effort. Eventually, learning a new category may only involve combining existing features in an appropriate manner. The

\footnotetext{
${ }^{*}$ Corresponding author. Tel./fax: +48-22-625-7460.

E-mail addresses: mandziuk@alpha.mini.pw.edu.pl (J. Mańdziuk), shastri@icsi.berkeley.edu (L. Shastri).

${ }^{1}$ This research was performed while the author was visiting International Computer Science Institute and EECS Department, University of California at Berkeley, Berkeley, CA, USA thanks to the support of Fulbright Senior Scholarship No. 20895.
} 
approach promotes the sharing of learned features among a number of categories and also alleviates the well-known catastrophic interference problem. We present promising results of applying the ICL approach to the unconstrained handwritten digit recognition problem, based on a spatio-temporal representation of patterns. (C) 2002 Elsevier Science Inc. All rights reserved.

Keywords: Incremental class learning; Catastrophic interference problem; Supervised learning; Spatio-temporal representation; Pattern recognition; Handwritten digit recognition; Neural network

\section{Introduction}

The catastrophic interference problem [1-3] remains a significant impediment in building large, scalable learning systems based on neural networks. In its simplest form, the problem may be stated as follows: when a network trained to solve task A is subsequently trained to solve task B, it "forgets" the solution to task A. In other words, the network is unable to acquire new knowledge without destroying previously acquired knowledge structures. A seemingly simple solution to this problem is to retrain the network on a cumulative training set containing examples from all previously learned categories. However, for large-scale problems this approach is not practical.

Incremental training methods are especially important for the fast or realtime control problems (e.g. ATM traffic control). Due to the large amount of noisy on-line data the Multilayer Perceptron-type networks with relatively slow retraining schemes are not suitable for such tasks. While there exist neural network models with learning schemes not prone to catastrophic interference (e.g. ART-type networks [4]), their effectiveness in dealing with large-scale and noisy problem domains is still under research. Some promising results have been obtained for the hybrid neuro-fuzzy ART models, e.g. Fuzzy ARTMAP, FasArt or PROBART.

The incremental class learning (ICL) approach [5] attempts to address the catastrophic interference problem and at the same time offers a learning framework that promotes the sharing of previously learned knowledge structures. With respect to object recognition and classification problems, the approach may be summarized as follows: The system starts off with all the nodes and links it will ever have, but initially, it focuses on only a small number of categories. After it learns to recognize these categories, it tries to identify which of the features formed in the "hidden layers" play a critical role in the recognition of these categories. The system "freezes" these critical features by fixing their input weights. As a result, they cannot be obliterated by subsequent learning. These frozen features, however, can participate in structures that are learned subsequently to recognize other categories. As the system learns to 
recognize more and more categories, it is hoped that the set of features will gradually stabilize and eventually, learning a new category will primarily consist of combining existing features in novel ways.

The paper is organized as follows. In Section 2, the proposed ICL approach is described and its relation to some of the existing incremental learning methods is discussed. Section 3 presents computer simulation results of the ICL for the handwritten digit recognition (HDR) problem. Conclusions and directions for future work appear in Section 4.

\section{Incremental class learning}

The ICL approach is a supervised learning procedure for neural networks that can be described as follows:

- Subproblems are learned incrementally.

- Structures playing a critical role in solving a subproblem are frozen.

- The above structures are available for subsequent learning.

- Solutions to subproblems are combined in an appropriate manner to solve the complete problem.

The success of the approach depends on four key factors. First, it should be possible to decompose the problem into subproblems in an effective manner. Second, the learning algorithm must develop relatively sparse (compact) structures to solve a subproblem. Third, it should be possible to identify features that play a critical role in solving a particular subproblem so that such features may be frozen. Fourth, it should be possible to combine solutions to subproblems whereby frozen features are shared among various solutions.

The proposed method can be viewed as a member of the general class of constructive algorithms since the functional structure of the network is constructed incrementally during learning by freezing appropriate nodes and links. There is, however, a significant difference between most constructive approaches and ICL. Typically, constructive methods (e.g. Cascade-Correlation [6] or Upstart Algorithm [7]) start with a minimal network and expand its structure by adding new nodes and links in order to minimize the overall network's error. In our approach, the network starts off with all the nodes and links it will ever have. Consequently, the representational capacity of the whole network is available right from the very start of the learning process (as is the case of backpropagation nets). This in turn allows more efficient search for the winning nodes in the competitive learning process.

The ICL method also shares some common features with modular approaches (e.g. [8-10]) in that both approaches make use of problem decomposition. In modular approaches, however, the subproblems are learned 
independently by separate modules, and then the solutions to the subproblems are combined to yield the solution for the initial problem. In the ICL method, subproblems are not learned independently and the structures learned for solving one subproblem are available for solving subsequent subproblems. Thus, unlike the modular approach, ICL allows considerable sharing of structure across subnetworks. Consequently, in case of the ICL, the resulting knowledge representation is expected to be more compact and less redundant compared to modular approaches.

The ICL approach resonates with the notion of competitive learning (cf. $[11,12])$ and also lifelong learning (cf. $[13,14]$ ) wherein learning new tasks becomes relatively easier when the number of tasks that have already been learned increases.

The current system implementation does not involve any relearning. However, it is possible to include some form of interleaved learning [2] within the ICL paradigm.

\section{ICL application to HDR problem}

In this section we present the application of the proposed ICL method to the HDR problem. The main objective of the work reported here is to show the efficacy of the proposed learning scheme in the context of a non-trivial and real-world problem domain involving noisy data. While we strive for a solid recognition performance, it is not our objective to develop a state-of-the-art HDR system. Such systems achieve higher recognition rates by incorporating sophisticated and specialized post- and pre-processing steps that are extrinsic to the core recognition and learning methodology.

First we briefly describe the spatio-temporal representation of patterns that was used in computer simulations. Then we present the architecture of the neural networks system used to implement the ICL method, and discuss experimental results that provide evidence for the effectiveness of the ICL approach in solving the classification task.

\subsection{Spatio-temporal representation of patterns}

In the spatio-temporal representation used in this work (cf. [9]) a two-dimensional static pattern is converted into a time-varying sequence of signals by sliding a window over the pattern (see Fig. 1). Doing so converts the spatial dimension of the pattern along the direction of scan into a temporal dimension. We will refer to the direction of scan as the temporal axis. At time $t=0$, the window is positioned at the beginning of the pattern, and for each subsequent $t$ it moves by one pixel column along the temporal axis. At each window position all nodes in the first hidden layer receive the input signal generated exactly by 


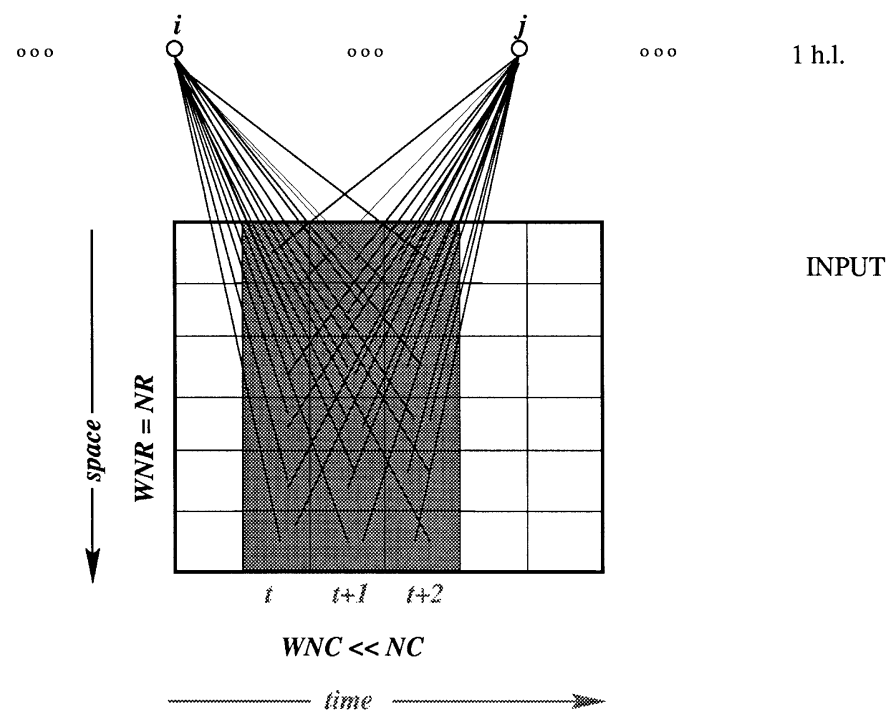

Fig. 1. Spatio-temporal pattern representation. A two-dimensional input pattern is converted into a sequence of signals generated by a window sliding over the pattern along the temporal axis. At each window position (at each time $t$ ) all nodes in the first hidden layer (1hl) receive the normalized signal generated by the part of the pattern which is currently covered by the window. $N R$ and $N C$ denote the number of rows and columns, respectively, in the input pattern, $W N R$ and $W N C$ denote the number of rows and columns, respectively, in the window being moved along the pattern.

the part of the pattern covered by the window. Note that since the width of the window along the temporal axis is greater than 1, the areas covered by neighboring windows overlap.

Advantages of the spatio-temporal representation: The proposed representation has some important advantages over the two-dimensional spatial representation. First, it ensures shift invariance along the temporal axis. Since the window generates input signals only when the actual content of the pattern is reached, the generated input sequence is independent of the location of the pattern along the temporal axis. Second, the spatio-temporal representation provides a natural framework for dealing with patterns of arbitrary extent along the temporal axis. Finally, the spatio-temporal representation simplifies a model's architecture. Usually, the extent of local features along the temporal direction is much smaller than the extent of the pattern itself. Therefore, the sliding window mechanism allows detection of local features with a much smaller number of links between the input layer and the first (hidden) layer of a network. For a detailed discussion of the advantages of the spatio-temporal approach please refer to [9]. Several other ways of representing off-line handwritten characters are discussed in [15]. 


\subsection{System architecture}

The system is composed of two modules operating simultaneously and independently on the input data. One module performs scanning of the input pattern along columns and the other one along rows - Fig. 2. Each module is a feed-forward neural network with two hidden layers and an output layer. In the testing phase, an additional output layer is used to combine evidence from the two modules, and to generate the final output of the system. The only difference between the modules is the direction of scan. Therefore, for the sake of brevity, the detailed description of the system will, henceforth, be based only on the column scan module (CSM).

The CSM is composed of the input layer, two hidden layers and the moduleoutput layer.



Fig. 2. System overview. In the training phase the system is composed of two independently operating modules - CSM and RSM which scan the input pattern along columns and rows, respectively. Each module is a feed-forward neural network composed of the input layer, two hidden layers and the module-output layer (denoted by "module-output" in the figure). In the testing phase the additional output layer is placed above the modules to combine evidence from both of them to produce the final output of the system. 
Input layer: In the input layer of the CSM, a sliding window of size $W N R \times W N C,(W N R=N R, W N C \ll N C$, where $N R$ and $N C$ denote numbers of rows and columns in an input pattern, respectively) moves from left to right along the temporal axis - one pixel-column at a time.

First hidden layer (1hl): Each node in $1 \mathrm{hl}$ is fully connected to the sliding window in the input layer with random initial weights. The role of the $1 \mathrm{hl}$ nodes is to find the representation of local features pertaining to the training patterns. These nodes are not tied to a particular class.

Second hidden layer (2hl): Higher level features composed of lower level features formed in $1 \mathrm{hl}$ are represented in this layer. Each node in $1 \mathrm{hl}$ is fully connected to nodes in $2 \mathrm{hl}$ with random initial weights. The role of a $2 \mathrm{hl}$ node is to find a complex feature pertaining to a specific class.

Module-output layer $(\mathrm{m}-\mathrm{ol})$ : The module-output layer is composed of $K$ nodes - one per class - where $K$ denotes the number of classes in the training set. The final response of the module is expressed in this layer. At the beginning of the training process the connections between $2 \mathrm{hl}$ and the $\mathrm{m}$-ol are extremely weak. The appropriate connections are strengthened during the learning process.

\subsubsection{Terminology and notation}

The training set is composed of $K$ classes $C_{k}, k=0, \ldots, K-1$, of binary $\{0,1\}$ patterns. Each class $C_{k}$ is composed of $M$ exemplars each of which is a matrix of $N R$ rows and $N C$ columns. The items in the training set will be referred to as $X_{m}^{k}[p][q], m=1, \ldots, M, p=0, \ldots, N R-1, q=0, \ldots, N C-1$.

Let $w_{1}[r][c][i]$ denote the weight between the $[r][c]$ element in the input window and the $i$ th node in the $1 \mathrm{hl}$, let $w_{2}[i][j]$ denote the weight between the $i$ th $1 \mathrm{hl}$ node and the $j$ th $2 \mathrm{hl}$ node, and let $w_{3}[i][j]$ denote the weight between the $i$ th 2 hl node and the $j$ th output node.

Moreover, let $F R_{1}[k]$ and $F R_{2}[k]$ denote sets of frozen nodes for class $C_{k}$ in the $1 \mathrm{hl}$ and the 2hl, respectively, and let $I_{1}^{r}[n], I_{2}^{r}[n]$, and $I_{3}^{r}[n]$, for $r=t r$ and $t s$ denote input activations to the $n$th node in the $1 \mathrm{hl}$, the $2 \mathrm{hl}$ and the m-ol, in the training and testing phases, respectively. Similarly, let $O_{1}^{r}[n], O_{2}^{r}[n]$, and $O_{3}^{r}[n]$ stand for the respective output activations of the $n$th node in the $1 \mathrm{hl}$, the $2 \mathrm{hl}$ and the m-ol, respectively in the training $(r=t r)$ and testing $(r=t s)$ phases.

Finally, let $H 1 S I Z E$ and $H 2 S I Z E$ denote the numbers of nodes in the $1 \mathrm{hl}$ and the $2 \mathrm{hl}$, respectively.

\subsubsection{Training phase}

Learning overview: First, all weights between input and $1 \mathrm{hl}$ nodes and between $1 \mathrm{hl}$ and $2 \mathrm{hl}$ nodes are initialized with small random values and then normalized so that the sum of squares of incoming weights of all $1 \mathrm{hl}$ and $2 \mathrm{hl}$ nodes equals 1 . Initially the weights on connections between the $2 \mathrm{hl}$ and the 
m-ol nodes are also set close to zero. The following pseudo-code presents the learning overview:

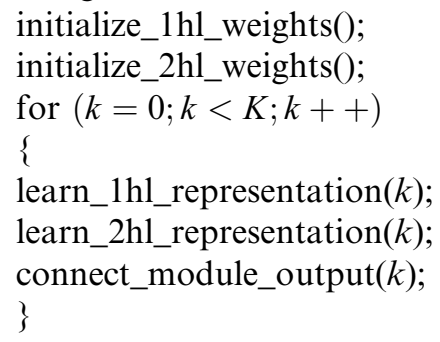

\subsubsection{Learning the 1 hl feature representation of a class}

The following discussion assumes that the system is currently learning the $k$ th class, $0 \leqslant k<K$.

The learning of $1 \mathrm{hl}$ features for class $k$ occurs as a result of the repeated presentation of all patterns of class $k$. The presentation of a pattern $X_{m}^{k}$ during a given epoch involves the following steps:

- the input window is positioned over a part of the image,

- activation within the window is normalized,

- a node in the $1 \mathrm{hl}$ with the highest input activation - called the winner - is found,

- its incoming weights are updated and normalized,

- the window is moved one step in the direction of scan and the above steps repeated until the image is fully scanned.

Window positioning: A window of $W N R$ rows and $W N C$ columns is initially positioned at the extreme left of the pattern (in the experiments reported here, $W N R$ was set equal to $N R$ ). Thereafter, the window shifts right, one step at a time, until the image is fully scanned.

Normalizing window's activation: $Y_{m}^{k}$, the activity resulting from the image at a given step of the scan is obtained by normalizing the image pixel values under the window in the following way:

$$
\begin{aligned}
& Y_{m}^{k}[r][t+c]:=\frac{X_{m}^{k}[r][t+c]}{\sqrt{\sum_{p=0}^{p=W N R-1} \sum_{q=0}^{q=W N C-1} X_{m}^{k}[p][t+q]}}, \\
& \quad r=0, \ldots, W N R-1, t=\text { scan step }, c=0, \ldots, W N C-1 .
\end{aligned}
$$

Finding the winner: Each node $i$ in the 1 hl computes its input activation $I_{1}^{\operatorname{tr}}[i](t)$

$$
I_{1}^{t r}[i](t)=\sum_{r=0}^{W N R-1} \sum_{c=0}^{W N C-1} Y_{m}^{k}[r][t+c] \cdot w_{1}[r][c][i]
$$


and the winner, i.e. the node whose weight vector is closest to $Y_{m}^{k}$ is found: ${ }^{2}$

$$
\text { winner }=\underset{i=0, \ldots, H 1 S I Z E-1}{\arg \max } I_{1}^{t r}[i](t) .
$$

Updating the winner's weights: The change in the weights of a 1 hl node depends on the degree of match between the node and window's content. The weight change procedure consists of the following three cases:

Case $(\alpha)$ : The winning node has a high degree of match with the window's content, but the node has already been frozen during the learning of some prior class $j$. That is,

$$
\sum_{r=0}^{W N R-1} \sum_{c=0}^{W N C-1} Y_{m}^{k}[r][t+c] \cdot w_{1}[r][c][\text { winner }]>\theta_{\text {shar }}
$$

and $\exists j<k:$ winner $\in F R_{1}[j]$. In this case, the weights of the winner are not changed. Note that, the winner has a high match with the current pattern, and hence, will automatically be shared between classes $k$ and $j$ (and any other classes with which the winner has a high degree of match). $\theta_{\text {shar }}$ is a predefined (high) threshold value for sharing nodes between classes.

Case $(\beta)$ : The winning node has a low degree of match with the window's content, and the node has already been frozen during the learning of some prior class $j$. That is,

$$
\sum_{r=0}^{W N R-1} \sum_{c=0}^{W N C-1} Y_{m}^{k}[r][t+c] \cdot w_{1}[r][c][\text { winner }] \leqslant \theta_{\text {shar }}
$$

and $\exists j<k:$ winner $\in F R_{1}[j]$. In this case, the unfrozen node that best matches the window's content is found and its weights are changed as in case $\gamma$ below.

Case $(\gamma)$ : The winning node has not been frozen during the learning of prior classes. That is, $\forall j<k$ : winner $\notin F R_{1}[j]$.

In this case the weights of the winner are updated as follows

$$
\begin{aligned}
& \Delta w_{1}[r][c][\text { winner }]:=\frac{\eta\left(Y_{m}^{k}[r][t+c]-w_{1}[r][c][\text { winner }]\right)}{\log (\text { mass }[\text { winner }])}, \\
& \text { mass }[\text { winner }]:=\operatorname{mass}[\text { winner }]+1,
\end{aligned}
$$

where $\operatorname{mass}[i], i=0, \ldots, H 1 S I Z E-1$ is the "inertia" of node $i$ and $\eta$ is a predefined learning rate. ${ }^{3}$ A node's inertia increases each time it is a winner, and hence, nodes tend to develop into stable feature detectors. A node's inertia is reset (set to 3) at the beginning of each training epoch.

\footnotetext{
${ }^{2}$ Recall that both the incoming weights' vector and input window's vector are normalized.

${ }^{3}$ In the experiments presented in the paper $\eta$ was set to 0.05 .
} 
Intuitively, the term mass refers to the inertia or resistance of a node to weight changes. As the node develops into a more stable feature detector its mass (inertia) increases and it becomes more resistive to changes in its incoming weights.

Normalizing winner's incoming weights: The winning node's incoming weights are normalized in the following way

$$
\begin{gathered}
w_{1}[r][c][\text { winner }]:=\frac{w_{1}[r][c][\text { winner }]}{\sqrt{\sum_{p=0}^{p=W N R-1} \sum_{q=0}^{q=W N C-1}\left(w_{1}[p][q][\text { winner }]\right)^{2}}}, \\
r=0, \ldots, W N R-1, c=0, \ldots, W N C-1 .
\end{gathered}
$$

Freezing relevant nodes: All the $1 \mathrm{hl}$ nodes, that became winners in the last epoch form the set of features, $F R_{1}[k]$, associated with class $C_{k}$ in the 1 hl. All of these nodes are frozen.

\subsubsection{Learning the class representation in the $2 \mathrm{hl}$}

The learning of complex features for class $k$ in the $2 \mathrm{hl}$ also occurs as a result of the repeated presentation of patterns of class $k$ over several epochs. In each epoch, for each pattern $X_{m}^{k}$, the following steps are performed:

- 1 hl representation of a pattern is found,

- some number of winners are found in the $2 \mathrm{hl}$,

- winners' weights are updated,

- winners' weights are normalized.

Finding 1 hl representation of a pattern: For each window position $t$, each $1 \mathrm{hl}$ node $i$ calculates its incoming activation $I_{1}^{t r}[i](t)$. These activations are stored in the auxiliary memories $M E M_{i}, i=0, \ldots, H 1 S I Z E-1$ (see Fig. 3).

After the scanning of the pattern is completed, the 1 hl representation of the pattern is defined by the set of nodes with the highest input activations across the layer, for each instance $t$. In other words, $W I N_{m}^{k}$, the representation of pattern $X_{m}^{k}$ in $2 \mathrm{hl}$ is defined as:

$$
W I N_{m}^{k}=\bigcup_{t} \underset{i=0, \ldots, H 1 S I Z E-1}{\arg \max } \operatorname{MEM}_{i}(t) .
$$

Output activations of the $1 \mathrm{hl}$ nodes are defined as:

$$
O_{1}^{t r}[i]= \begin{cases}\frac{1}{\sqrt{\left|W N_{m}^{k}\right|}} & \text { if } i \in W I N_{m}^{k} \\ 0 & \text { otherwise. }\end{cases}
$$

Finding winners in the $2 \mathrm{hl}$ : Having the $1 \mathrm{hl}$ representation of the pattern activated, the input activations to the $2 \mathrm{hl}$ nodes are calculated

$$
I_{2}^{t r}[p]=\sum_{i=0}^{H 1 S I Z E-1} O_{1}^{t r}[i] \cdot w_{2}[i][p], \quad p=0, \ldots, H 2 S I Z E-1
$$




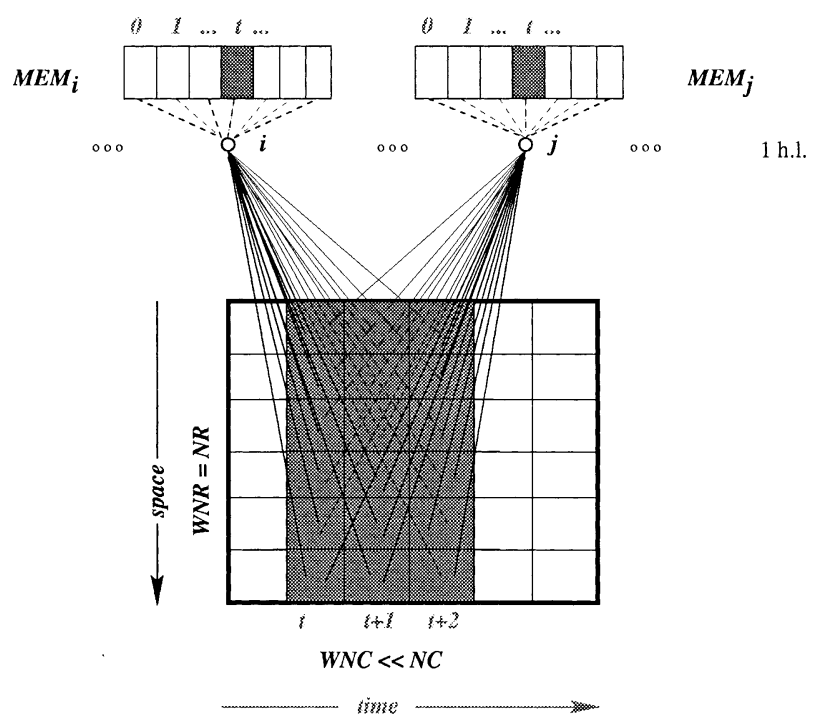

Fig. 3. Training of the $2 \mathrm{hl}$. First the $1 \mathrm{hl}$ representation of the pattern is found. Each $1 \mathrm{hl}$ node $i$, at each window position, stores its incoming activation in the auxiliary memory $M E M_{i}$. The $1 \mathrm{hl}$ pattern representation is defined as the set of nodes with the highest activations for all instances $t-$ one "winning" node per one window position.

and the first $\rho$ nodes with the highest input activations are chosen - denoted by $\operatorname{win}_{1}, \ldots$, win $_{\rho}$ in the equations below. ${ }^{4}$ Namely,

$$
\operatorname{win}_{s}=\underset{p=0, \ldots, H 2 S I Z E-1, p \neq \operatorname{win}_{1}, \ldots, p \neq \text { win }_{s-1}, p \notin F R_{2}}{\arg \max } I_{2}^{t r}[p], \quad s=1, \ldots, 5 .
$$

Note, that the winning nodes are chosen only among not yet frozen nodes. Therefore, there is no sharing of nodes between classes in the $2 \mathrm{hl}$.

Updating winners' weights: Weights between the $1 \mathrm{hl}$ nodes and the winning nodes in the $2 \mathrm{hl}$ are updated in the following way

$$
\begin{gathered}
\Delta w_{2}[i]\left[\operatorname{win}_{s}\right]:=\frac{\psi\left(O_{1}^{t r}[i]-w_{2}[i]\left[\text { win }_{s}\right]\right)}{\text { scale }[s]}, \quad i=0, \ldots, H 1 \text { SIZE }-1, \\
s=1, \ldots, 5, \text { scale }=[1,2,2,3,3],
\end{gathered}
$$

where $\psi$ is a predefined learning rate. ${ }^{5}$ The degree of weights change is scaled by coefficient scale based on the "winning position" of the winning node. Scaling of learning coefficients adds some flexibility in assigning $2 \mathrm{hl}$ nodes to

\footnotetext{
${ }^{4}$ In the current implementation, $\rho$ is set to 5 .

${ }^{5}$ In the experiments presented in the paper $\psi$ was set to 0.05 .
} 
particular $1 \mathrm{hl}$ representations in the learning process. Some nodes develop into precise detectors of $1 \mathrm{hl}$ representations, while others detect "average" $1 \mathrm{hl}$ representations of patterns from class $k$.

Normalizing winners' weights: The incoming weights of the winning nodes are normalized in a manner similar to that used for normalizing the incoming weights of the $1 \mathrm{hl}$ nodes.

$$
\begin{gathered}
w_{2}[i]\left[\operatorname{win}_{s}\right]:=\frac{w_{2}[i]\left[\operatorname{win}_{s}\right]}{\sqrt{\sum_{j=0, \ldots, H 1 S I Z E-1}\left(w_{2}[j]\left[\operatorname{win}_{s}\right]\right)^{2}}}, \\
i=0, \ldots, H 1 S I Z E-1, \quad s=1, \ldots, 5 .
\end{gathered}
$$

Freezing relevant nodes: All $2 \mathrm{hl}$ nodes, which became winners in the last epoch form a representation $F R_{2}[k]$ of class $C_{k}$ in the $2 \mathrm{hl}$. All of them are frozen.

\subsubsection{Building connections between the $2 \mathrm{hl}$ and the $\mathrm{m}$-ol}

Once the $2 \mathrm{hl}$ training phase for class $k$ is completed the connections between all $2 \mathrm{hl}$ nodes which were frozen for that class and a node representing class $k$ in the $\mathrm{m}$-ol are strengthened to be equal to 1 (Fig. 4).

\subsubsection{Pruning the network}

Once the training process is completed the network structure is minimized by pruning irrelevant nodes and links. All nodes (along with their respective links) in the $1 \mathrm{hl}$ and the $2 \mathrm{hl}$, which were not frozen by any class are deleted. Pruning operation has no impact on system's performance. The main reason for pruning is to make the system more compact, and hence, computationally efficient. However, pruning should not be done if the system is going to be ex-

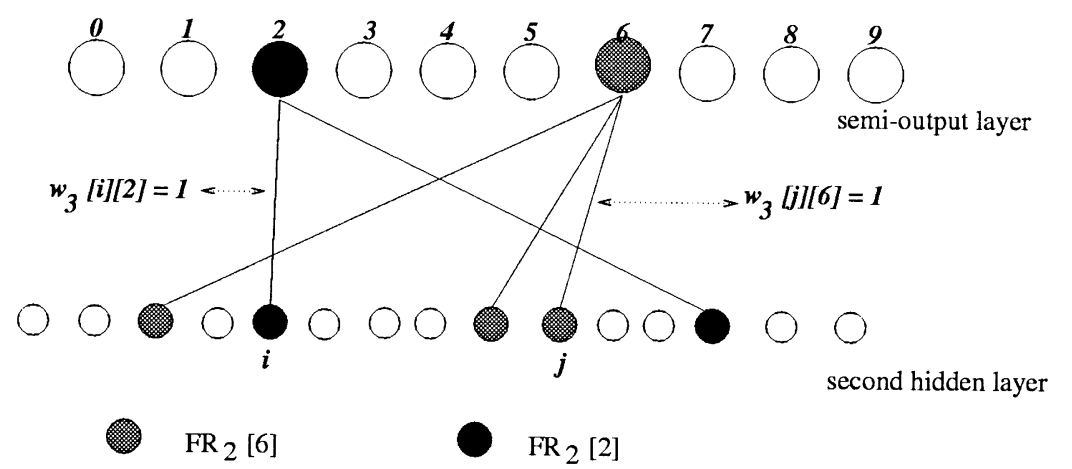

Fig. 4. Building connections between the $2 \mathrm{hl}$ and the $\mathrm{m}$-ol layer. For the currently learned class $k$, connection weights between nodes in the $2 \mathrm{hl}$ that represent class $k$ (nodes from the set $F R_{2}[k]$ ) and the node representing class $k$ in the mo-l are strenghtened to be equal to 1 . 
posed to other learning tasks in the future since unfrozen nodes may be required for capturing new features created during subsequent learning.

\subsubsection{Testing phase}

In the testing phase (Fig. 5), the unknown pattern is presented to the input layer, and scanned left-to-right by the CSM, and top-to-bottom by the row scan module (RSM), using a scanning window. While a pattern is being scanned, each node in $1 \mathrm{hl}$ maintains a cumulative "winning activation". In other words, at each scan step $t$, the node $i$ in the 1 hl that receives the highest input activation in that step increases its cumulative input activation $I_{1}^{t s}[i]$ by the current activation value $\operatorname{MEM}_{i}(t)$. More formally,

$$
I_{1}^{t s}[i]=\sum_{t \in T(i)} \operatorname{MEM}_{i}(t), \quad i=0, \ldots, H 1 S I Z E-1,
$$

where

$$
T(i)=\left\{t: \underset{m=0, \ldots, H 1 \text { SIZE }-1}{\arg \max } \operatorname{MEM}_{m}(t)=i\right\} .
$$

Output activations from the $1 \mathrm{hl}$ nodes are normalized

$$
O_{1}^{t s}[i]=\frac{I_{1}^{t s}[i]}{\sqrt{\sum_{j=0}^{H 1 S I Z E-1}\left(I_{1}^{t s}[j]\right)^{2}}}, \quad i=0, \ldots, H 1 S I Z E-1 .
$$

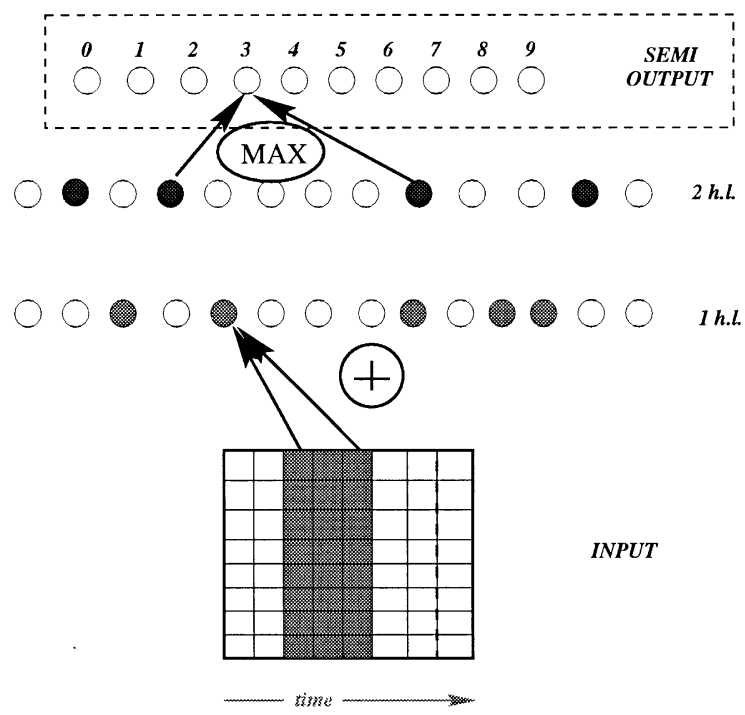

Fig. 5. Overview of the testing procedure - CSM. See text for description. 
The input activations to all $2 \mathrm{hl}$ nodes are calculated

$$
I_{2}^{t s}[i]=\sum_{j=0}^{H \text { ISIZE }-1} w_{2}[j][i] \cdot O_{1}^{t s}[j], \quad i=0, \ldots, H 2 \text { SIZE }-1
$$

and the set $W I N_{2}$ composed of $p$ nodes with the $p$ highest input activations is defined ( $p$ is a system parameter). Then the output activations from $2 \mathrm{hl}$ nodes are calculated in the following way:

$$
O_{2}^{t s}[i]= \begin{cases}I_{2}^{t s}[i] & \text { if } i \in W N_{2}, \\ 0 & \text { otherwise. }\end{cases}
$$

Input to each of the m-ol nodes is calculated as the maximum input among the $2 \mathrm{hl}$ nodes contributing to this $\mathrm{m}$-ol node. Namely,

$$
I_{3}^{t s}[k]=\max _{i=0, \ldots, H 2 S I Z E-1}\left(w_{3}[i][k] \cdot O_{2}^{t s}[i]\right), \quad k=0, \ldots, K-1 .
$$

Finally,

$$
O_{3}^{t s}[k]=I_{3}^{t s}[k], \quad k=0, \ldots, K-1 .
$$

The evidence from the CSM and the RSM in the testing phase is combined in the final output layer (Fig. 6) in the following way:

$$
O U T[k]=\alpha \cdot O U T^{c}[k]+\beta \cdot O U T^{r}[k],
$$

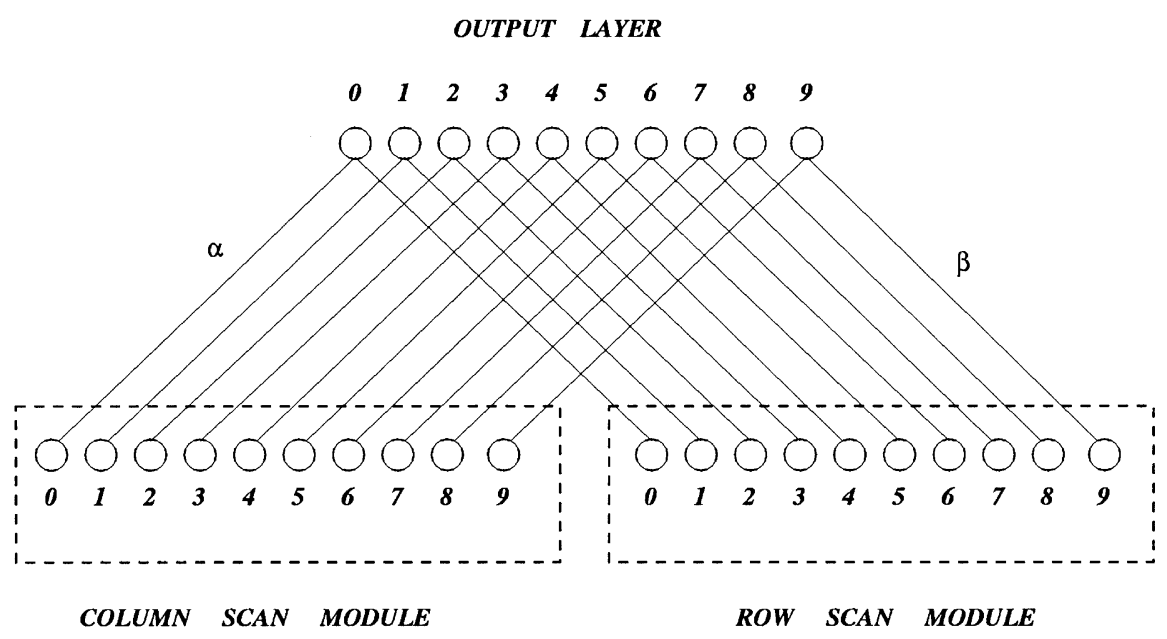

Fig. 6. Testing phase. Each output layer node's value is a linear combination of respective evidence from CSM and RSM. All links between the m-ol nodes in the CSM and in the RSM and the output layer nodes are equal to $\alpha$ and $\beta$, respectively. 
where $O U T^{c}[k], O U T^{r}[k]$ denote outputs from the $k$ th nodes in the moduleoutput layers in the CSM and RSM, respectively, and OUT $[k]$ is the input (and output) activation of the $k$ th node in the final output layer.

Several schemes for combining classifiers in the HDR domain has been proposed in the literature $[16,17]$. Some of them does not involve any knowledge about the nature of the classifiers (e.g. voting) others incorporate additional information (e.g. Dempster-Shafer, mixture of experts). A linear combination (weighted sum) classifier used in this paper assumes independence of the two combined classifiers on one hand, but at the same time allows adding some confidence (parameters $\alpha$ and $\beta$ ) in the quality of their classifications. Based on our experience with HDR [9] we put more confidence into column scanning than into row scanning $(\alpha>\beta)$.

\subsection{Experimental results}

The efficacy of proposed ICL approach was tested on the HDR problem by computer simulations of the neural network architecture and learning scheme described above. The set of binary $\{0,1\}$ patterns representing handwritten digits was extracted from the USPS CEDAR database. ${ }^{6}$ The database is composed of approximately 2400 handwritten zip codes collected from pieces of mail. Both five and nine digit zip codes are included. The zip codes were broken down into individual digits by making linear divisions between consecutive digits without removing stray marks or extended strokes. The data used in our experiment was composed of 10 classes $C_{k}, k=0, \ldots, 9$ (one class per digit) each of which contained 600 training and 600 test patterns $X_{m}^{k}[][], m=0, \ldots, 599,600, \ldots, 1199$. An additional set of 100 patterns was used for cross-validation.

\subsubsection{Preprocessing}

Size normalization: Patterns, originally of various sizes, were normalized [18] by resizing from rectangle to square, sampling with a regular interval, comparing the normalized sum of activations of pixels in the surrounding square with the threshold value $(=0.5)$ and, if greater or equal than the threshold, setting the corresponding pixel in the resulting image to 1 , or setting it to 0 , otherwise. The size of patterns after normalization was equal to $17 \times 17$. Some exemplar digits before and after size normalization are presented in Fig. 7.

\footnotetext{
${ }^{6}$ The database is officially known as the "United States Postal Service Office of Advanced Technology Handwritten ZIP Code Database (1987)" and was made available for research by the Office of Advanced Technology, United States Postal Service.
} 
(a)
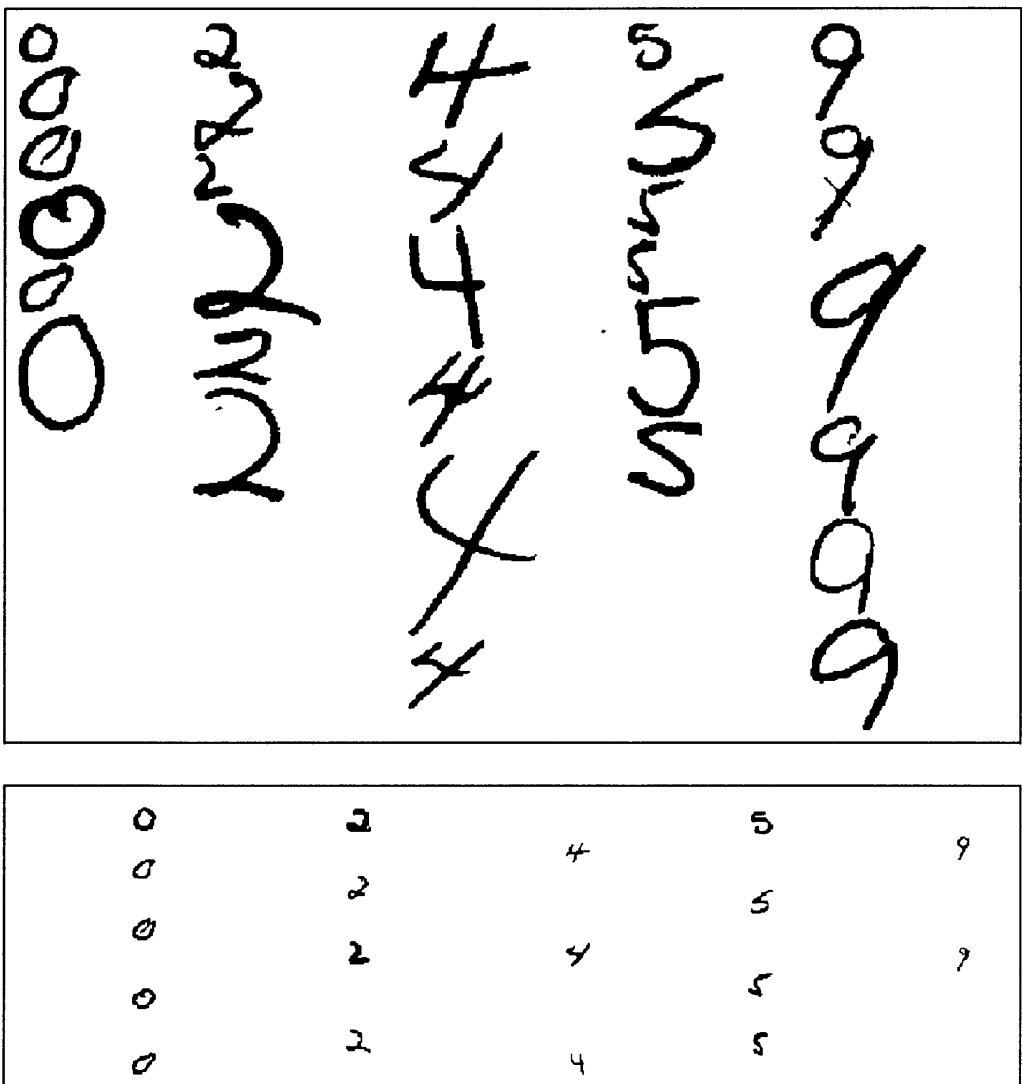

5

2

4

2

5

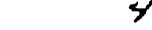

5

9

2

4

5

0

$z$

5

9

4

2

$S$

9

$G$

$y$

9

(b)

Fig. 7. Examples of training patterns before (a) and after (b) size normalization. Figures have the same scale.

Other preprocessing techniques: Several other preprocessing techniques are commonly used in the statistical or neural-nets based HDR methods [15,17]. Examples of such techniques include low pass filtering, skeletonization, skew 


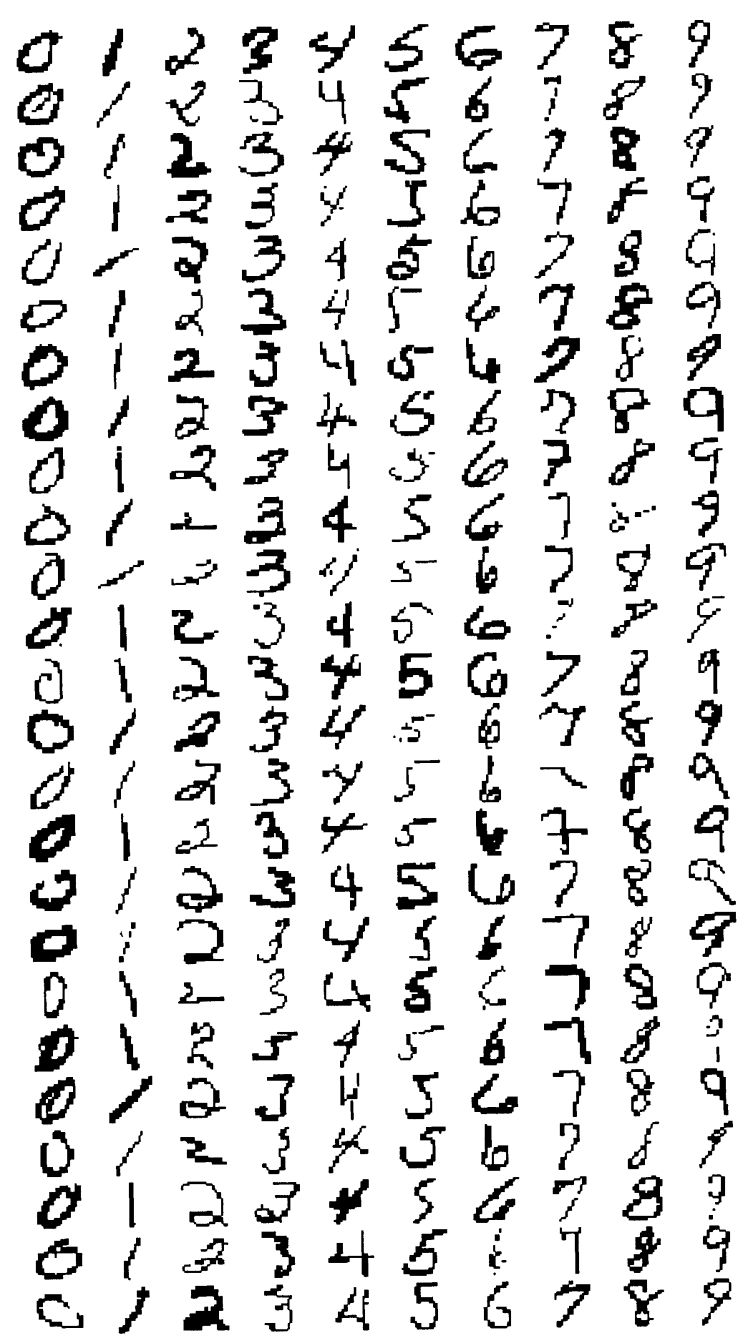

Fig. 8. Examples of training patterns from the CEDAR database, after size normalization, used in the simulations.

normalization, thinning, line regularization, discarding "empty" patterns, and more.

Therefore, for the fair comparison with other published HDR results it is worth noting that except size normalization no other preprocessing techniques were applied to the data. Consequently, preprocessed digits preserved high variations in thickness and skewness. Additional examples of size-normalized input patterns are presented in Fig. 8 . 


\subsubsection{Defining system's parameters}

Most of system's parameters (e.g. the learning rate, inertia coefficients, sizes of layers, numbers of winners, numbers of training epochs) were chosen based on pilot simulations, as well as on intuition and past experience of the authors. General guidelines for the number of nodes in the first and the second hidden layer are to allow sufficient nodes in the 1 hl to make the repertoire of potential features as extensive and comprehensive as possible. Moreover, the process of feature development in the $1 \mathrm{hl}$ should be continued long enough to allow the development of reliable feature detectors. In contrast, learning in the $2 \mathrm{hl}$ can be performed relatively easily, since the main objective here is the aggregation of already recognized $1 \mathrm{hl}$ features into class representatives (these can be viewed as exemplars or templates). Moreover, learning in the $2 \mathrm{hl}$ can be performed relatively fast if proper feature detectors have been learned in the 1 hl.

\subsubsection{Simulation results}

One of the observations from preliminary simulations was that the order in which classes are presented during learning has some influence on the quality of results. This effect is due to the fact that order of presentation influences which features are learned first. This in turn shapes the rest of the learning process since these primarily developed features are available for representations of subsequent classes.

Therefore, in the final system setup four modules - two CSMs and two RSMs were used. Combining evidence from four (instead of two) independent modules resulted in greater flexibility and reliability in the system's behavior. One CSM and one RSM used the ascending order of classes, that is $\{0,1,2,3,4,5,6,7,8,9\}$. The order of learning was chosen independently for the remaining two modules $(\{9,4,1,6,5,7,3,8,2,0\}$ for the other CSM, and $\{9,7,6,5,0,4,3,8,2,1\}$ for the other RSM). In the final output layer both CSMs were treated equally (with the same weight). Similarly, the respective links between each of the RSMs and the final output layer were pairwise equal. In general, links between CSMs and the final output layer were different from those between RSMs and the final output layer. Hence, the input to node $k$ in the final output layer, denoted by $O U T[k]$ in (22) was equal to

$$
O U T[k]=\alpha \cdot O U T^{c 1}[k]+\beta \cdot O U T^{r 1}[k]+\alpha \cdot O U T^{c 2}[k]+\beta \cdot O U T^{r 2}[k],
$$

where $O U T^{c 1}[k], O U T^{c 2}[k], O U T^{r 1}[k]$ and $O U T^{r 2}[k]$ denote outputs from the respective module-output layers of the four modules. The final output of the system was the class number corresponding to the maximum value OUT $[k], k=0, \ldots, 9$. 
The number of nodes in each layer was established empirically based on some preliminary simulations, and for each of the four modules was equal to 5000,1200 and 10 for the $1 \mathrm{hl}$, the $2 \mathrm{hl}$ and the m-ol, respectively.

Among the 20,000 and 4800 available nodes in the $1 \mathrm{hl}$ and the $2 \mathrm{hl}$ of the four modules, respectively, the system froze 10,442 and 1840 nodes, respectively, during the training phase. The system effectively used about $2.1 \times 10^{6}$ out of about $2.5 \times 10^{7}$ available links.

As described above, learning in the $1 \mathrm{hl}$ and in the $2 \mathrm{hl}$ was based on multiple presentation of all exemplars from the currently learned class. The numbers of training epochs per class were equal to 50 and 10 for the $1 \mathrm{hl}$ and the $2 \mathrm{hl}$, respectively. Based on preliminary simulations we observed that increasing $1 \mathrm{hl}$ training epochs to 75 or 100 had no particular impact on the system efficiency. On the other hand, reducing the number of epochs below 30 resulted in degraded system performance.

Several alternative schemes for computing the network's output were considered. Two of these are described below.

Raw performance: The output was computed under the following condition: - $\alpha=\beta=1$,

- $p=1$ (i.e. one winner was selected in the $2 \mathrm{hl}$ in the testing phase in each of the four modules).

The system's performance was $97.87 \%$ on the training set and $92.55 \%$ on the test set.

Enhanced performance: We experimented with a small set of alternate values for $\alpha, \beta$ and $p$ and observed that the system's performance could be improved by differentially weighting the responses of the row and column networks. For example, the choice of

- $\alpha=0.9, \beta=1.3$,

- $p=8$ for CSMs and $p=3$ for RSMs

improved the system's performance on the test set to $93.13 \%$.

Results with rejection: In the experiments reported thus far, a pattern was rejected if the ratio between the second and the first best choices in the final output layer was relatively high, that is if

$$
k_{1}:=\underset{k}{\arg \max }\{O U T[k]\} \quad \text { and } \quad k_{2}:=\underset{k \neq k_{1}}{\arg \max }\{O U T[k]\},
$$

then

$$
\frac{O U T\left[k_{2}\right]}{O U T\left[k_{1}\right]}>\text { threshold } \Rightarrow \text { reject pattern. }
$$

The plot of system's performance with various levels of rejection is presented in Fig. 9. 


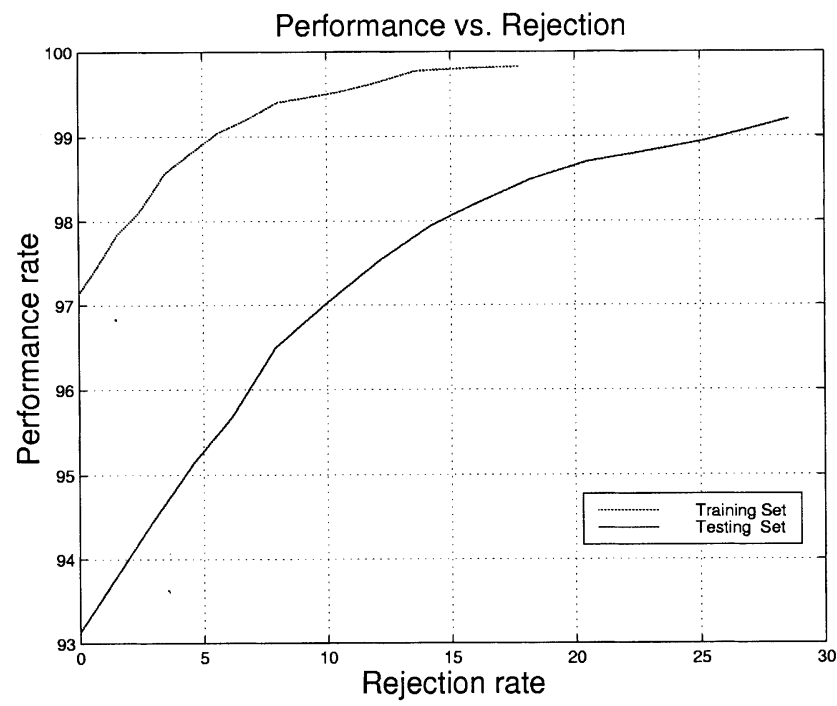

Fig. 9. A plot of system's performance on the training and test sets with rejection.

The performance on the test set exceeded $94 \%, 95 \%, 96 \%$ and $97 \%$ with $1.9 \%, 4.4 \%, 6.9 \%$ and $9.9 \%$ rejection, respectively. The performance on the training set exceeded $98 \%$ and $99 \%$ with $2.1 \%$ and $5.4 \%$ rejection, respectively.

Misclassified patterns: In the experiment without rejection, the performance on the test set was equal to $93.13 \%$, that is, 412 out of 6000 patterns were incorrectly classified. Some of these misclassified patterns were highly ambiguous. Examples of misclassified patterns are presented in Fig. 10. The confusion matrix for the test set is presented in Table 1.

Several conclusions can be drawn from analysis of Table 1. In general the observations are qualitatively not surprising due to some shape resemblance between exemplars of particular (handwritten) digits. For example any two classes from the set $\{3,5,8\}$ are often misclassified each with other. Similarly class 1 is often confused with class 4 and vice versa.

Significance of experimental results: The simulation results obtained for the CEDAR USPS database suggest that the ICL approach outlined in this paper is an effective and plausible method of solving classification problems involving a large number of classes.

In evaluating the results it may be appropriate to note that our goal was to evaluate the efficacy of the ICL learning scheme on a real-life and non-trivial problem domain involving noisy data. The above results suggest that the ICL approach in quite effective in solving the HDR problem. It seems reasonable to assume that the performance of the system could be improved further by using larger training sets and performing more sophisticated preprocessing (e.g. skew 

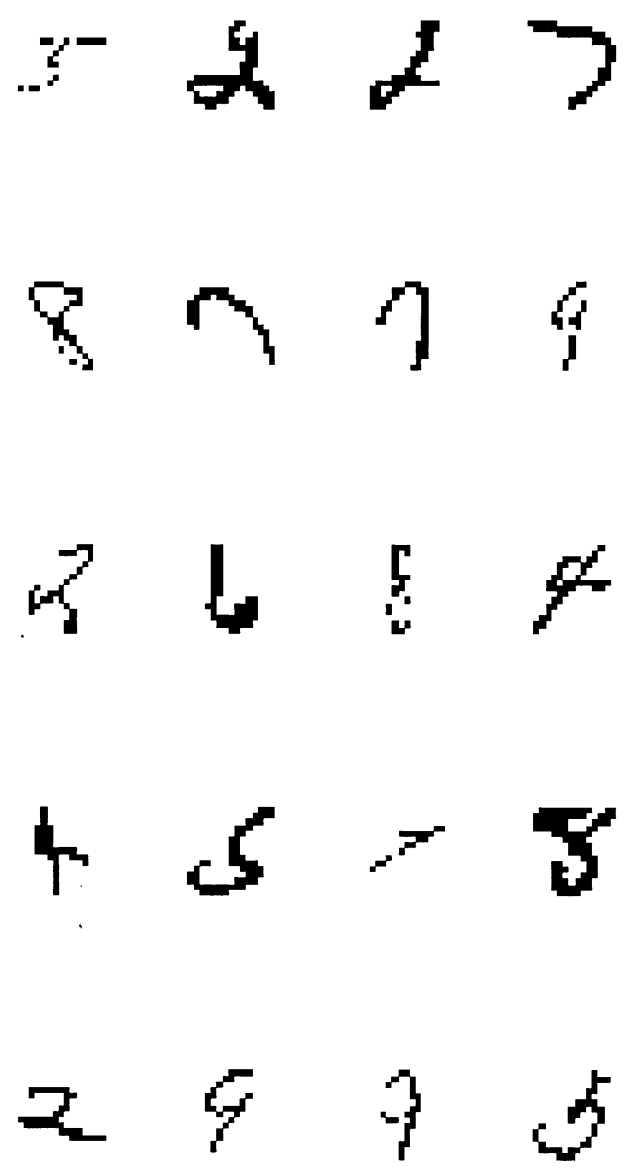

Fig. 10. Some examples of misclassified patterns from the test set.

normalization and skeletonization). We did not pursue these possibilities since we are not interested in developing a state-of-the-art HDR system.

The performance of the ICL based HDR system was compared with that of nearest neighbor classifier (NNC) using the training patterns used in the ICL experiment. The NNC is widely used as a benchmark for other classifiers mainly because in most applications it proved to provide reasonably good classifications. Its other advantages include easy implementation and problem independence [17].

The recognition rate of $\mathrm{NNC}$ on the test set was $86.36 \%$.

The quality of ICL results is comparable to the other published results on the CEDAR database. For example in [19] an accuracy of $95.42 \%$ with $0 \%$ rejection on a test set composed of 2007 digits was reported and in [9] a 96.0\% 
Table 1

Misclassification table for the test set in the experiment without rejection

\begin{tabular}{rrrrrrrrrrrr}
\hline- & $\mathbf{0}$ & \multicolumn{1}{c}{$\mathbf{1}$} & \multicolumn{1}{c}{$\mathbf{2}$} & \multicolumn{1}{c}{$\mathbf{3}$} & \multicolumn{1}{c}{$\mathbf{4}$} & \multicolumn{1}{c}{$\mathbf{5}$} & $\mathbf{6}$ & $\mathbf{7}$ & $\mathbf{8}$ & $\mathbf{9}$ & $\sum$ \\
\hline $\mathbf{0}$ & - & 2 & 1 & 1 & 0 & 1 & 6 & 0 & 15 & 0 & 26 \\
$\mathbf{1}$ & 0 & - & 0 & 0 & 10 & 0 & 0 & 1 & 3 & 2 & 16 \\
$\mathbf{2}$ & 7 & 4 & - & 11 & 3 & 4 & 1 & 14 & 9 & 4 & $\mathbf{5 7}$ \\
$\mathbf{3}$ & 0 & 0 & 6 & - & 0 & 13 & 0 & 10 & 15 & 4 & 48 \\
$\mathbf{4}$ & 0 & 22 & 5 & 0 & - & 1 & 0 & 1 & 3 & 13 & 45 \\
$\mathbf{5}$ & 0 & 2 & 4 & 27 & 0 & - & 0 & 1 & 7 & 3 & 44 \\
$\mathbf{6}$ & 4 & 9 & 9 & 1 & 5 & 8 & - & 1 & 7 & 0 & 44 \\
$\mathbf{7}$ & 1 & 10 & 2 & 0 & 11 & 8 & 0 & - & 2 & 12 & 46 \\
$\mathbf{8}$ & 7 & 9 & 1 & 16 & 3 & 9 & 0 & 0 & - & 3 & 48 \\
$\mathbf{9}$ & 0 & 7 & 1 & 1 & 17 & 0 & 0 & 10 & 2 & - & 38 \\
$\sum$ & 19 & $\mathbf{6 5}$ & 29 & 57 & 49 & 44 & 7 & 38 & $\mathbf{6 3}$ & 41 & 412 \\
\hline
\end{tabular}

In each row of the table, the number of misclassifications of the respective digit (the first value in a row) with all other classes is presented. The rightmost column presents a sum of all misclassifications for this digit. For example, digit 3 was misclassified with digit 5 in 13 cases, and digit 5 with digit 3 in 27 cases.

accuracy with no rejection and $99.0 \%$ accuracy with $9.5 \%$ rejection was reported on the test set containing 2700 images. Knerr et al. [20] have reported an accuracy of $95.8 \%$ with $2.4 \%$ rejections for the pixel-based representation of digits and an improved performance of $97.47 \%$ with $1 \%$ rejections for the feature-based representation.

In the context of the above results the accuracy of $93.13 \%$ with $0 \%$ rejection in case of totally unconstrained images seems significant.

Possible directions for improvement: The system's performance may be improved by refining the ICL approach and we are pursuing several possibilities. These include:

- selection of multiple winning nodes in $2 \mathrm{hl}$,

- propagation of negative evidence from the $1 \mathrm{hl}$ nodes to the $2 \mathrm{hl}$ nodes, and

- the fine tuning of shared (frozen) nodes by allowing such nodes to modify their input weights - albeit with very high inertia.

Based on pilot simulations we believe that the fine tuning of shared nodes can improve the system's performance.

\subsubsection{Stabilization of feature set in the $1 \mathrm{hl}$}

One of the expected properties of the system is the asymptotic stabilization of the feature set in the $1 \mathrm{hl}$. One would expect that after the network has learned a sufficient number of classes, learning would become relatively easy and primarily involve the use of features from previously learned tasks.

The numbers of recruited nodes per class for the two orders used in the training of CSM are presented in Table 2. These results suggest that such asymptotic behavior was not observed in the current set of experiments and 
Table 2

Numbers of nodes recruited by subsequent classes in the CSM based training in two different orders of classes

\begin{tabular}{lcclcc}
\hline Class & New nodes & All nodes & Class & New nodes & All nodes \\
\hline $\mathbf{0}$ & 176 & 176 & $\mathbf{9}$ & 352 & 352 \\
$\mathbf{1}$ & 60 & 111 & $\mathbf{4}$ & 292 & 497 \\
$\mathbf{2}$ & 416 & 564 & $\mathbf{1}$ & 72 & 238 \\
$\mathbf{3}$ & 390 & 648 & $\mathbf{6}$ & 243 & 464 \\
$\mathbf{4}$ & 312 & 612 & $\mathbf{5}$ & 348 & 704 \\
$\mathbf{5}$ & 346 & 856 & $\mathbf{7}$ & 125 & 511 \\
$\mathbf{6}$ & 255 & 778 & $\mathbf{3}$ & 419 & 919 \\
$\mathbf{7}$ & 107 & 527 & $\mathbf{8}$ & 563 & 1254 \\
$\mathbf{8}$ & 546 & 1265 & $\mathbf{2}$ & 332 & 1077 \\
$\mathbf{9}$ & 291 & 951 & $\mathbf{0}$ & 174 & 790 \\
& 2899 & & & 2920 & \\
\hline
\end{tabular}

For each CSM learning order, columns from left to right denote: the class number, number of new nodes for this class, number of all (new and shared with previous classes) nodes for the class.

subsequent classes still recruit many new nodes (e.g. digit 8). It appears that the number of classes will have to be increased beyond ten in order to observe the expected convergence of the feature set. In order to verify the hypothesis that there will be a gradual decrease in the number of nodes frozen for a new class we plan to extend the experiment to the set of all alphanumeric characters. The other possibility is to continue training the existing "trained system" on another handwritten digits database and see if there is significant overlap between the nodes frozen in response to the new set of exemplars of a given class and those frozen in response to the old set of exemplars of that class.

An interesting observation from Table 2 is that the overall number of nodes frozen by all classes is independent of the class order (the same holds for RSMs).

\section{Conclusions}

In this work we have proposed the ICL approach based on freezing relevant features and sharing common (similar) features among multiple classes. The ICL approach not only takes advantage of existing knowledge when learning a new problem, it also offers immunity from the catastrophic interference problem. Promising results obtained for the unconstrained HDR problem suggest that the approach may be a suitable framework for building large, scalable learning systems. We conjecture that the sharing of relevant features among classes will make subsequent learning more effective (easier and faster), especially when a new task exhibits a degree of similarity to previously learned tasks. Hence, the ICL approach is also the promise of dealing with large-scale 
and life-long learning. The above conjecture requires further experimental evaluation in other problem domains.

One of the key factors in the potential success of this approach is the ability to decompose a problem into subproblems. While such a decomposition may not be easily achievable for certain problems, many naturally occurring problem domains such as child language acquisition and development seem to have a structure that lends itself to problem decomposition. For example, children acquire a few basic nouns and verbs and master a few basic grammatical constructs before moving onto learning more complex nouns, verbs, and constructions [21].

Another important issue is the observed influence of the presentation order on the quality of results. Several other incremental learning systems (e.g. ART networks) suffer from the same problem. One possibility to alleviate this influence is combining several modules using different presentation orders as was done in this research. However, in general case this aspect requires more experimental and theoretical studies.

In future we plan to verify the efficacy of the ICL method in other problem domains and to work on developing more compact representations of learned tasks in 1hl, mainly by involving other criteria for the selection of frozen features. At the same time, we are interested in using alternate criteria for combining evidence from multiple network modules. Finally, we are also investigating effective methods for problem decomposition.

\section{References}

[1] M. McCloskey, N.J. Cohen, Catastrophic interference in connectionist networks: the sequential learning problem, in: G. Bower (Ed.), The Psychology of Learning and Motivation, Academic Press, New york, 1989, pp. 109-165.

[2] J.L. McClelland, B.L. McNaughton, R.C. O'Reilly, Why there are complementary learning systems in the hippocampus and neocortex: insights from the successes and failures of connectionist models of learning and memory, Technical Report: PDP.CNS.94.1, 1994.

[3] E. Pessa, M.P. Pennaand, Catastrophic interference in learning process by neural networks, in: Proc. of the ICANN'94, Sorrento, Italy, 1994, pp. 589-592.

[4] G.A. Carpenter, S. Grossberg, A massively parallel architecture for a self-organizing neural pattern recognition machine, Computer Vision Graphics and Image Processing 37 (1987) 54 115 .

[5] L. Shastri, Attribution learning as a solution to the catastrophic interference problem in learning with neural nets, Working Paper, International Computer Science Institute, 1994.

[6] S.E. Fahlman, C. Lebiere, The cascade-correlation learning architecture, in: D. Touretzky (Ed.), Advances in Neural Information Processing Systems, vol. 2, Morgan Kaufmann, Los Latos, CA, 1990, pp. 524-532.

[7] M. Frean, The upstart algorithm: a method for constructing and training feedforward neural networks, Neural Computation 2 (1990) 198-209. 
[8] A. Waibel, Consonant recognition by modular construction of large phonemic time-delay neural networks, in: D. Touretzky (Ed.), Advances in Neural Information Processing Systems, vol. 1, Morgan Kaufmann, Los Altos, CA, 1989, pp. 215-223.

[9] L. Shastri, T. Fontaine, Recognizing handwritten digit strings using modular spatio-temporal connectionist networks, Connection Science 7 (3) (1995) 211-235.

[10] P. Mitra, S. Mitra, S.K. Pal, Staging of cervical cancer using soft computing, IEEE Transactions on Biomedical Engineering 47 (7) (2000) 934-940.

[11] D. Rumelhart, D. Zipser, Feature discovery by competitive learning, Cognitive Science 9 (1985) $75-112$.

[12] T. Kohonen, Self-organized formation of topologically correct feature maps, Biological Cybernetics 43 (1982) 59-69.

[13] S. Thrun, T.M. Mitchell, Learning one more thing, Technical Report: CMU-CS-94-184, 1994.

[14] S. Thrun, Explanation Based Neural Network Learning. A Lifelong Learning Approach, Kluwer Academic Publishers, Boston, 1996.

[15] R. Plamondon, S.N. Srihari, On-line and off-line handwriting recognition: a comprehensive survey, IEEE Transactions on Pattern Analysis and Machine Intelligence 22 (1) (2000) 63-84.

[16] L. Xu, A. Krzyzak, C.Y. Suen, Methods for combining multiple classifiers and their applications in handwritten character recognition, IEEE Transactions on Systems, Man, and Cybernetics 22 (1992) 418-435.

[17] A.K. Jain, R.P.W. Duin, J. Mao, Statistical pattern recognition: a review, IEEE Transactions on Pattern Analysis and Machine Intelligence 22 (1) (2000) 4-37.

[18] H. Hou, Digital Document Processing, Wiley, New york, 1983.

[19] Y. Le Cun, B. Boser, J. Denker, D. Henderson, R. Howard, W. Hubbard, L. Jackel, Handwritten digit recognition with a back-propagation network, in: D. Touretzky (Ed.), Advances in Neural Information Processing Systems, vol. 2, Morgan Kaufmann, Los Altos, CA, 1990, pp. 396-404.

[20] S. Knerr, L. Personnaz, G. Dreyfus, Handwritten digit recognition by neural networks with single-layer training, IEEE Transactions on Neural Networks 3 (6) (1992) 962-968.

[21] M. Tomassello (Ed.), Beyond Names for Things: Young Children's Acquisition of Verbs, Lawrence Erlbaum, Hillsdale, NJ, 1995. 